\title{
Attitudes Towards Pediculosis Treatments in Teenagers
}

\author{
Deon V. Canyon*, Chauncey Canyon, Sami Milani and Rick Speare
}

Office of Public Health Studies, University of Hawaii at Manoa, 1960 East-West Rd, Biomed Building \#T103, Honolulu, HI 96822, USA

\begin{abstract}
Research on pediculosis has focused on treatment strategies and social aspects have been largely ignored. Pediculosis and its treatment in are associated with negative emotional responses while in developing countries pediculosis and its treatment may provide more an opportunity for positive social bonding. Attitudes to pediculosis have been proposed as important to successful control. Previous studies in Australia found that parents of primary school children say they treat pediculosis once it has been detected.

This study retrospectively investigated attitudes towards treatment in teenage high school students in an attempt to collect information from those afflicted rather than from parents. Only participants with a history of pediculosis were recruited from a high school in Western Australia and they were asked to complete an anonymous questionnaire. The sample contained 128 Grade 8 and 9 students, aged 13-15 years old with an even gender split. Negative feelings towards being treated for head lice were observed in $41.5 \%$ of males and $54.7 \%$ of females and $49.5 \%$ of Caucasians and $40 \%$ of Asians. Anti-treatment sentiment was expressed by $19.7 \%$ of males and $10.9 \%$ of females. Shampooing with and without combing were the most preferred treatments overall.

The results showed that $63.6 \%$ male and $52.7 \%$ female high school students were in favour of head lice treatments. This low percentage indicates that current treatments for head lice require improvement to be made more acceptable and that alternative treatments that are less unpleasant need to be developed. Strategies need to be explored to make treatment of pediculosis a more positive emotional experience.
\end{abstract}

Keywords: Combing, feelings, hair thickness, head lice, treatment,

\section{INTRODUCTION}

Pediculus humanus capitis De Geer, the insect ectoparasite that causes pediculosis, has evolved in close association with human hosts with archeological evidence extending back 9000 years [1]. Pediculosis in developed countries is mainly treated by application of liquid compounds to the hair. These typically have an insecticidal action and are often combined with use of a fine-toothed "nit" comb to physically remove lice and lice eggs [2]. Other less commonly used treatments include specialised combs that electrocute lice and equipment to kill lice using hot air $[3,4]$. Head lice repellents, although lacking studies to demonstrate efficacy, are being increasingly sought to prevent transmission [5]. Research on pediculosis has focused on treatment strategies and social aspects have been largely ignored.

A study of adults ( $91 \%$ female) on issues associated with treatment of pediculosis found a broad range of technological, biological and social issues [6]. Many respondents in this survey found commercial products and methods ineffective and difficult and had concerns about their safety. Trying several treatments was commonly

*Address correspondence to this author at the Office of Public Health Studies, University of Hawaii at Manoa, 1960 East-West Rd, Biomed Building \#T103, Honolulu, HI 96822, USA; Tel: (808) 956-6263;

E-mail: dcanyon@hawaii.edu reported. Pain associated with use of nit combs was also reported. Decades ago a society's attitude to pediculosis was proposed as important to successful control [7]. Previous studies indicated that most parents of primary school children in Australia say they treat pediculosis once detected $[8,9]$. This was also the case in Norway [10].

Some researchers propose that perhaps a more important role is played by certain social factors and individual behavior $[6,7,11,12]$. While chemical or natural treatments may succeed in eliminating lice, the lice-free period may often be short-lived. Reinfection from other infected people is almost guaranteed if associates of the treated person (and their associates) are not treated concurrently. The root cause of reinfection traces back to behavior and attitudes. Product concerns, treating children, blaming others for reinfection, stigma and social issues are major concerns of parents and carers $[11,12]$ that remain to be adequately investigated in the infected population. Work by Canyon and Speare showed that a significant degree of apathy was present in infected students and their parents [13, 14]. Certain key individuals (school students) who remained apathetic due to a lack of awareness or lack of symptoms or desire to avoid treatment were observed to serve as potent head lice carriers. In fact, the growing global prevalence of pediculosis may be partly a result of this attitude [13]. This study thus aimed to investigate the attitudes of secondary school children to head lice treatments that largely occurred during primary/ elementary school, with a view to providing more informat- 
ion on teenage attitudes to pediculosis that serve to diminish head lice control efforts.

\section{METHODS}

The site of this study was a multicultural school in Perth, Western Australia with around 1700 students from Grades 8 to 12. Consent letters and information sheets were sent home to the parents of 480 middle-school students from Grades 8 and 9. Eligible teenagers with parental consent were asked to complete an anonymous survey that they returned to the school for collection. Participation was thus based on voluntary self-selection and diagnosis of pediculosis was not confirmed. Only students who could recall being infected with head lice were included in the study. It is possible that this sampling strategy introduced non-participation/nonresponse bias which may have lead to differing results. This sampling strategy combined criterion based and convenience approaches, since the targeted participants are those who have direct experience coping with a head lice infestation (criterion) and they are accessible (convenience) [15]. The questionnaire requested basic non-identifying demographic information (grade, gender and hair details) followed by two open-ended questions:

How do you feel about getting treated for head lice with combs, shampoos and other treatments?

What do you feel are the worst and best treatments for head lice?

"Shampoos" referred to topical liquid treatments, including both shampoos and lotions. The computer software package SPSS 20 was used to analyze responses for salient themes that addressed the questionnaire focus. Crosstabulations with Chi-Square tests were employed where possible, but low cell frequencies meant that Uncertainty Coefficient Tests were often employed. Ethics approval H2954 was obtained from James Cook University Human Ethics Committee.

\section{RESULTS}

The response rate was $27.7 \%$ with 133 students out of 480 participating in the study and 128 surveys that were sufficiently complete to enable analysis (Table 1). However, since only students who had experienced pediculosis were eligible to participate, the true response rate cannot be determined.

In response to the question, "How do you feel about getting treated for head lice with combs, shampoos and other treatments?" negative experiences included: smell, disgust, phobia, annoyance, inconvenience, wasted time, 'I prefer having lice', and pain. Non-negative experiences included: 'good', 'I like it', and 'I don't mind'. Responses were divided into five categories: anti-process, anti-shampoo, anticomb, anti-treatment and pro-treatment, which included neutral responses.

Anti-Process: $20.3 \%$ of all participants had a problem with the process of lice treatment. Gender was significant with $12.1 \%$ of males and $32.7 \%$ of females being annoyed or disgusted with the treatment (Chi-Square $\mathrm{p}<0.01)$. Caucasian females were significantly more anti-process than males $(p<0.05)$. Race and hair-related variables were not significant.
Table 1. Frequencies of personal factors in children with pediculosis.

\begin{tabular}{|c|c|}
\hline Personal Factors & Frequency $(\%)$ \\
\hline Gender & 2 missing \\
\hline Male & $68(53.1)$ \\
\hline Female & $58(45.3)$ \\
\hline Current Grade $\widehat{\jmath} / q$ & 3 missing \\
\hline 8 & $38 / 28(30.4 / 22.4)$ \\
\hline 9 & $29 / 30(23.2 / 24.0)$ \\
\hline Grade during infection $\widehat{\top} / q$ & 10 missing \\
\hline 1 & $4 / 2(3.4 / 1.7)$ \\
\hline 2 & $5 / 6(4.2 / 5.1)$ \\
\hline 3 & $7 / 4(5.9 / 3.4)$ \\
\hline 4 & $7 / 9(5.9 / 7.6)$ \\
\hline 5 & $11 / 9(9.3 / 7.6)$ \\
\hline 6 & $11 / 12(9.3 / 10.2)$ \\
\hline 7 & $15 / 8(12.7 / 6.8)$ \\
\hline 8 & $7 / 1(5.9 / 0.8)$ \\
\hline Ethnicity $\pi / q$ & 4 missing \\
\hline Caucasian & $57 / 50(46.0 / 40.3)$ \\
\hline Asian & $10 / 7(8.1 / 5.6)$ \\
\hline Hair Thickness $\delta / q$ & 2 missing \\
\hline Thin & $10 / 12(7.9 / 9.5)$ \\
\hline Medium & $35 / 27(27.8 / 21.4)$ \\
\hline Thick & $23 / 19(18.3 / 15.1)$ \\
\hline Hair Color $3 / P$ & 2 missing \\
\hline Blond & $12 / 9(9.5 / 7.1)$ \\
\hline Brown & $41 / 43(32.5 / 34.1)$ \\
\hline Black & $15 / 6(11.9 / 4.8)$ \\
\hline Hair Length $\pi / q$ & 5 missing \\
\hline Short & $57 / 1(46.3 / 0.8)$ \\
\hline Shoulder & $8 / 26(6.5 / 21.1)$ \\
\hline Long & $2 / 29(1.6 / 23.6)$ \\
\hline
\end{tabular}

Anti-Shampoo: $11.7 \%$ of all participants had a problem with lice treatment shampoos. Gender was moderately significant with $4.5 \%$ of males and $20.0 \%$ of females disliking the smell (Chi-Square $\mathrm{p}<0.01$ ). When gender was broken down by ethnicity, females remained significantly more likely to be anti-shampoo than males (Uncertainty coefficient: Caucasians $\mathrm{p}<0.01, \mathrm{u}=0.062$; Asians $\mathrm{p}<0.05$, $\mathrm{u}=0.259$ ). Hair length significantly affected anti-shampoo sentiment among Caucasians with fewer shorthaired people $(2.2 \%)$ and more longhaired people $(22.2 \%)$ being antishampoo (Chi-square $\mathrm{p}<0.05$ ). Ethnicity, hair thickness and hair color were not significant. 
Anti-Combing: $10.9 \%$ of all participants had a problem with fine-toothed lice combs. Hair thickness was significant with $17.9 \%$ of thick-haired, $11.3 \%$ of medium-haired, and $0.0 \%$ of thin-haired people disliking the pain $(\mathrm{p}<0.05$, $\mathrm{u}=0.40$ ). Gender, ethnicity, hair color and length were not significant.

Anti-Treatment: $16.3 \%$ of participants explicitly expressed anti-treatment sentiment, but the only significant association was that people with thick hair were significantly less likely to be anti treatment $(\mathrm{p}<0.05, \mathrm{u}=0.034)$.

Pro-Treatment: $57.7 \%$ of participants explicitly stated that they were in favor of head lice treatments, but all study factors were not significant.

Participant responses to the question "What do you feel are the worst and best treatments for head lice?" are presented in Table 2.

With regard to the worst treatments for head lice, several significant associations were identified. Hair thickness was moderately significant $(\mathrm{p}<0.05, \mathrm{u}=0.071)$ with more medium-haired people saying 'doing nothing' (adjusted residual $[\mathrm{AR}]=1.9$ ), no thick-haired person saying that manual removal or cutting is worst (AR -1.8, -1.9), more medium-haired people saying cutting is worst $(\mathrm{AR}=2.0)$, and more thick haired people having no particular distaste for any treatments $(\mathrm{AR}=2.0)$ (Figs. 1, 2). Hair length was more significant $(\mathrm{p}<0.01, \mathrm{u}=0.082)$ with, more longhaired people saying 'doing nothing' is worse $(\mathrm{AR}=2.2)$, more shorthaired people disliking combs $(\mathrm{AR}=1.6)$, more shoulder-lengthhaired people saying they had no preference $(A R=4.1)$. Hair color was not significantly related to worst treatment.

\section{DISCUSSION}

Some children are known to under-report infections to parents and some parents are known to avoid treating their children $[13,14]$. The aim of this study was to take this further and retrospectively investigate how teenage students felt about getting treated for head lice with combs, shampoos and other methods in primary/elementary school, and which treatments they considered worst and best.

\section{How Do you Feel About Getting Treated for Head Lice?}

Negative feelings towards being treated for head lice were observed in almost half of males, females, Caucasians and Asians with specific anti-treatment sentiment expressed by a fifth of males and a tenth of females and pro-treatment sentiment was expressed by over half of females and half of males. These feelings were displayed for the entire treatment experience and particular methods, such as application of topical liquids and combing. These results have implications, primarily for the industries providing these goods and the parents and guardians attempting to use them to treat their children. For manufacturers, smelly liquid treatments can be modified to have a more appealing odor to females and finetoothed lice combs can be made available in different widths to suit different hair thickness to reduce pain.

Few thick-haired students were anti-treatment compared to a fifth of thin and medium hair types. This may be due to thick-haired individuals being less sensitive or more used to painful treatments or more cognizant of the need for treatment. Hair length was not associated with being more or less anti-treatment. Hair thickness was not strongly associated with being more or less pro-treatment. Shorter

Table 2. Head lice treatments considered worst and best by female and male Caucasians and Asians.

\begin{tabular}{|c|c|c|c|c|c|}
\hline & & \multicolumn{2}{|c|}{ Caucasian $(\%)$} & \multicolumn{2}{|c|}{ Asian (\%) } \\
\hline & & Female & Male & Female & Male \\
\hline \multirow[t]{7}{*}{ Worst Treatments } & Doing Nothing & 9.6 & 9.6 & 23.1 & 7.7 \\
\hline & Combing & 11.7 & 18.1 & 7.7 & 30.8 \\
\hline & Shampoo/Herbal & 12.8 & 14.9 & 0.0 & 0.0 \\
\hline & Manual Removal & 3.2 & 2.1 & 0.0 & 7.7 \\
\hline & Cutting Hair & 1.1 & 3.2 & 0.0 & 7.7 \\
\hline & No Preference & 9.6 & 4.3 & 7.7 & 7.7 \\
\hline & Totals & 48 & 52 & 38 & 62 \\
\hline \multirow[t]{10}{*}{ Best Treatments } & Doing nothing & 0.0 & 1.0 & 0.0 & 0.0 \\
\hline & Combing & 5.1 & 8.2 & 0.0 & 8.3 \\
\hline & Combing + Shampoo/Conditioner & 14.3 & 11.2 & 8.3 & 8.3 \\
\hline & Shampoo/Conditioner & 10.2 & 15.3 & 0.0 & 16.7 \\
\hline & Chemical/Herbal Treatment + Others & 9.2 & 8.2 & 16.7 & 16.7 \\
\hline & Manual Removal & 0.0 & 2.0 & 0.0 & 0.0 \\
\hline & Cutting Hair & 2.0 & 2.0 & 0.0 & 8.3 \\
\hline & Electro Combing & 1.0 & 3.1 & 0.0 & 0.0 \\
\hline & No Preference & 6.1 & 1.0 & 8.3 & 8.3 \\
\hline & Totals & 48 & 52 & 33 & 67 \\
\hline
\end{tabular}




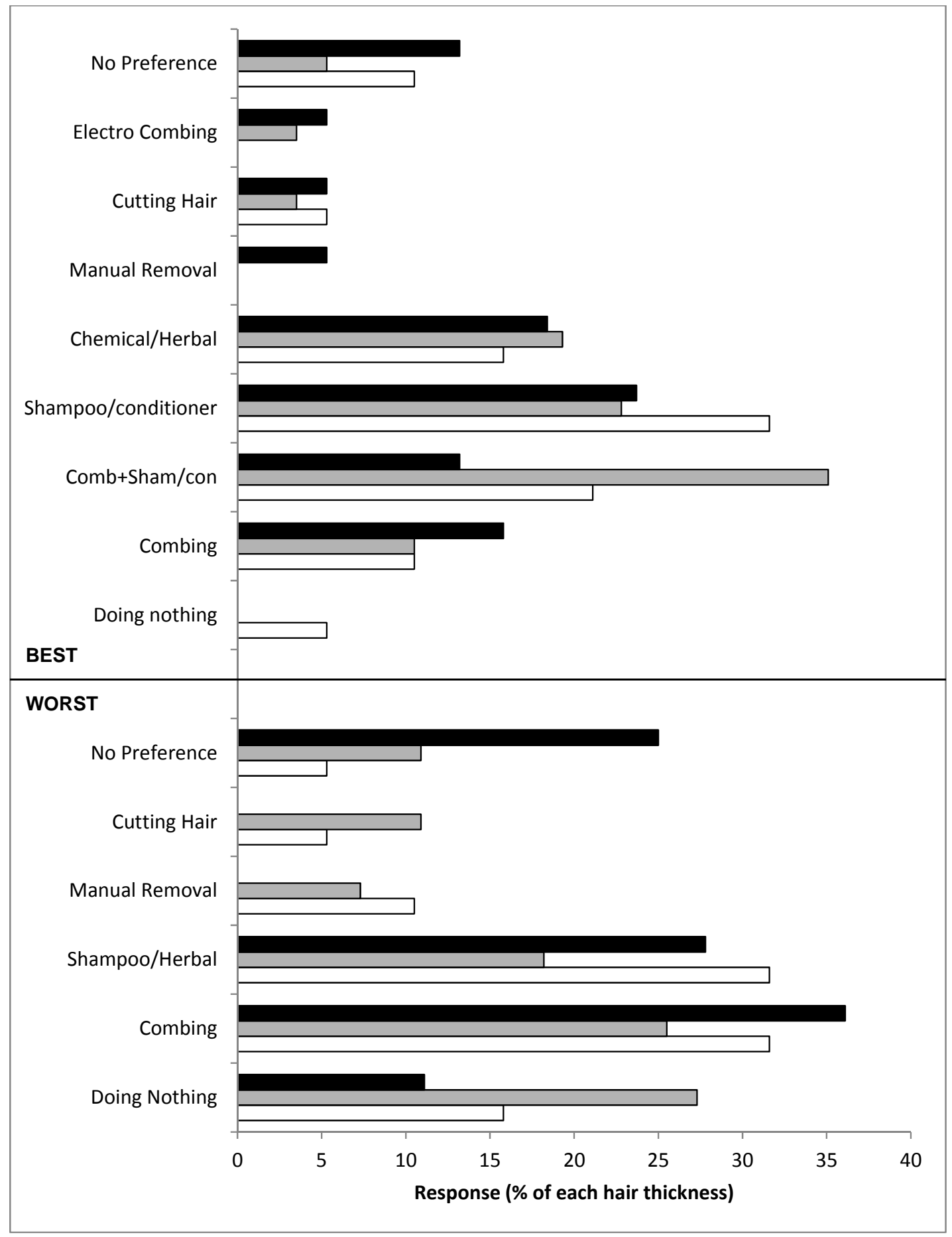

Fig. (1). Treatments considered to be the Best and Worst by participants with thin ${ }^{\square}$, medium ${ }^{\square}$ and thick $\square$ hair.

hair lengths were associated with being more pro-treatment, but not significantly so.

A small proportion of thin haired people (5.3\%) and shorthaired people $(2 \%)$ indicated that doing nothing was the best treatment. These children are at risk of becoming sources of head lice by concealing infection and avoiding treatment.

\section{What Do you Feel are the Worst and Best Treatments for} Head Lice?

Caucasian students thought combing and topical liquids were the worst treatments while Asian students thought doing nothing and combing were the worst. This response revealed more of the social environment in which head lice infections exist. For example, a fifth of Caucasian and a third 


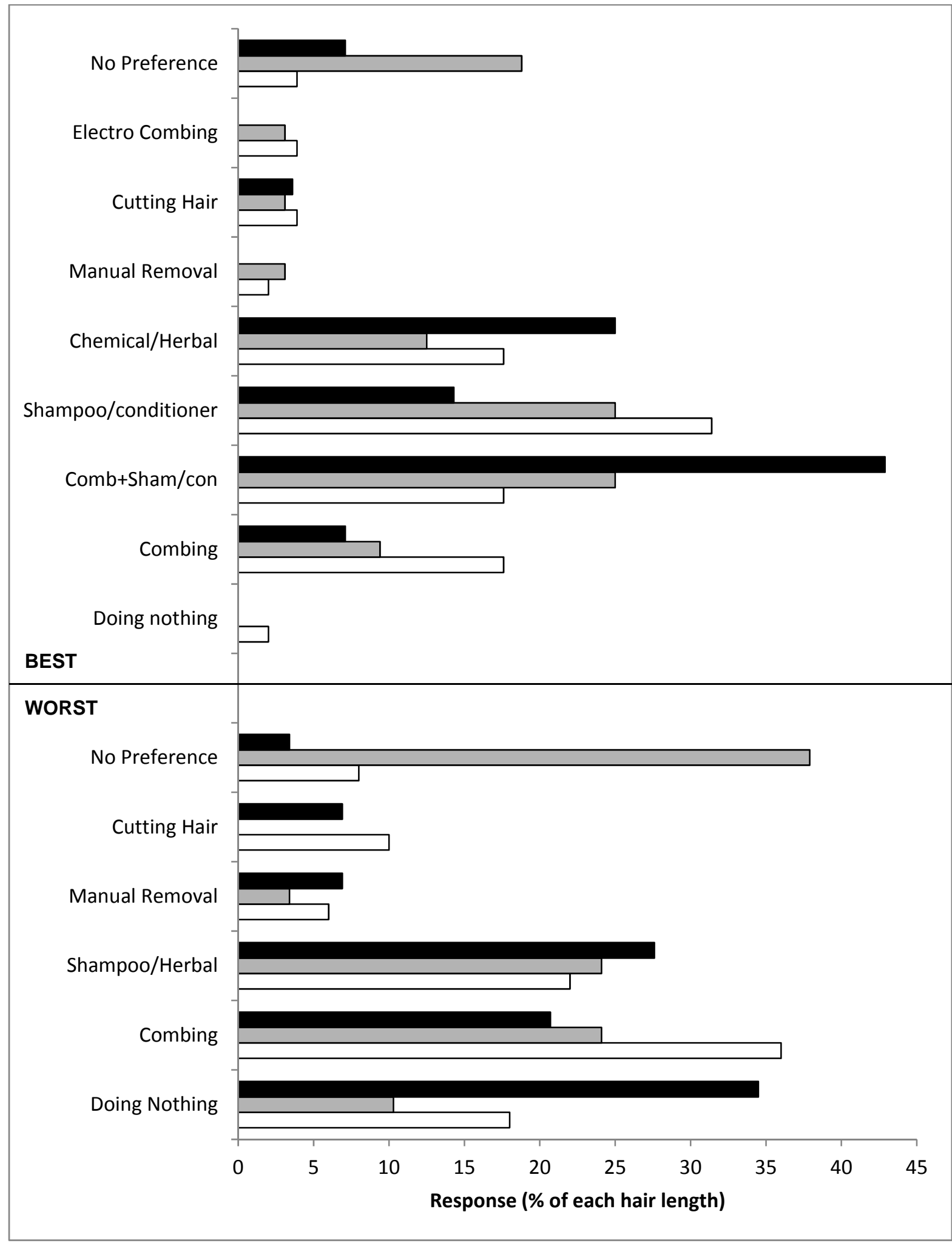

Fig. (2). Treatments considered to be the Best and Worst by participants with short ${ }_{\square} \quad$, shoulder ${ }_{\square} \quad$ and long $\square$ length hair.

of Asians said that doing nothing was the worst possible treatment. Given that there are a number of treatment options available, this was not expected. It may be that some students are aware of other infected students who chose to do nothing about their head lice infections or it may be that these students were often left untreated by their parents.
Hair thickness and length were significantly related to the stated worst treatments. It was confirmed that thick-haired students had a higher dislike than other hair types for combing. This supports the idea that this more painful method may be responsible for them concealing head lice infections. A higher proportion of shorthaired students 
disliked combing, so it may be the case that shorthaired students are combed more frequently or more roughly than longhaired students.

A higher proportion of longhaired students were against doing nothing. This may be because head lice eggs are difficult to remove and they stay on long hair for many months. As the hair grows out, the empty eggs from old infections become more visible. Thus long-term head lice infections in longhaired students are more likely to be detected visually by casual observers, which would result in social discomfort. Overall, the application of topical liquid treatments with and without combing were the most preferred treatments, which indicates a certain degree of tolerance in most teenagers to standard methods.

\section{LIMITATIONS}

Since most respondents wrote about pediculosis they had experienced in Grades 4 to 7, their recall would have been reasonable. A minority, who recalled episodes of pediculosis more than 3 years previously, may have been less accurate.

\section{CONFLICT OF INTEREST}

The authors confirm that this article content has no conflict of interest.

\section{ACKNOWLEDGEMENTS}

Declared none.

\section{PATIENTS CONSENT}

Informed Consent was given to the Author by the patients in respect of the clinical trials conducted.

\section{REFERENCES}

[1] Mumcuoglu KY, Zias J. Head lice, Pediculus humanus capitis (Anoplura: Pediculidae), from hair combs excavated in Israel and dated from the first century B.C. to the eighth century A.D. J Med Entomol 1988; 25: 545-7.

[2] Speare R, Canyon DV, Cahill C, Thomas G. Comparative efficacy of two nit combs in removing head lice (Pediculus humanus var capitis) and their eggs. Int J Dermatol 2007; 46(12): 1275-8.

[3] O'Brien E. Detection and removal of head lice with an electronic comb: zapping the louse. J Pediatr Nurs 1998; 13(4): 265-6.

[4] Goates BM, Atkin JS, Wilding KG, et al. An effective nonchemical treatment for head lice: a lot of hot air. Pediatrics 2006; 118(5): 1962-70

[5] Canyon DV, Speare R. A comparison of botanical and synthetic substances commonly used to prevent head lice (Pediculus humanus var. capitis) infestation. Int J Dermatol 2007; 46: 422-26.

[6] Parison J, Speare R, Canyon DV. Uncovering family experiences with head lice: the difficulties of eradication. Open Dermatol $\mathrm{J}$ 2008; 2: 9-17.

[7] Maunder B. Attitude to head lice--a more powerful force than insecticides. J R Soc Health 1985; 105(2): 61-4.

[8] Speare R, Buttner P. Prevalence of head lice in a primary school in Australia and implications for control. Int J Dermatol 1999; 38 : 285-90.

[9] Counahan M, Andrews R, Speare R. Reliability of written parental reports of head lice in their children. Med J Aust 2005; 182(3): 137-8.

[10] Rukke BA, Birkemoe T, Soleng A, Lindstedt HH, Ottesen P. Head lice in Norwegian households: actions taken, costs and knowledge. PLoS One 2012; 7(2): e32686.

[11] Parison J, Canyon DV. Head lice and the impact of knowledge, attitudes and practices - a social science overview. In: Management and control of head lice infestations. Bremen: UNI-MED Verlag AG, 2010; 103-9.

[12] Parison J, Speare R, Canyon DV. Head lice: the feelings people have. Int J Dermatol 2013; 52(2): 169-71.

[13] Canyon DV, Speare R. Clinical decision support: Dermatology: Pediculosis. Wilmington, DE: Decision Support in Medicine LLC, 2012.

[14] Canyon DV, Speare R. Head lice transmission and risk factors. In: Heukelbach J, Ed. Management and control of head lice infestations. Bremen: UNI-MED Verlag AG, 2010; 34-40.

[15] Patton MQ. Qualitative research \& evaluation methods, $3^{\text {rd }}$ Ed. Thousand Oaks, CA: Sage, 2002. 